\title{
Numéro spécial : Sciences et technologies innovantes pour l'amélioration des performances sportives de haut niveau
}

Dans trois ans se tiendront les Jeux Olympiques et Paralympiques de Paris 2024. Autant dire que c'est demain. Pour cet événement phare du sport mondial, l'objectif fixé par le Président de la République est de voir la France rentrer dans le top 5 des nations en matière de nombre de médailles olympiques, tous sports confondus. Pour mémoire, la France s'est située au $8^{\mathrm{e}}$ rang lors des derniers jeux de Tokyo 2021 après avoir occupé la $7^{\mathrm{e}}$ place à Rio 2016 et Londres 2012 et le $5^{\mathrm{e}}$ rang à Atlanta 1996.

L'objectif est donc d'inverser une tendance de recul des performances du sport français. Ceci s'inscrit dans une dynamique d'accueil des Jeux, généralement favorable aux sportifs du pays hôte mais aussi une condition de Jeux réussis. Dans ce contexte, l'État a mobilisé dans le cadre des plans d'Investissement d'Avenir (PIA3), 20 millions d'euros pour soutenir la recherche française au service de la performance sportive de très haut niveau. Il s'agit d'un effort sans précédent qui donne à nos laboratoires de recherche et Fédérations Sportives une double responsabilité.

Responsabilité pour ne pas dire «obligation» de résultats, évidemment, pour faire en sorte que les athlètes français soient les plus performants possible... et gagnent davantage de médailles que lors des éditions précédentes. C'est le préliminaire explicite formulé en introduction du Plan Prioritaire de Recherche.

Mais cet objectif n'est que la partie émergée de l'iceberg, celle du court terme, qui permettra, chacun l'espère, des succès plus nombreux lors de cette olympiade. Mais le second enjeu au moins aussi important, la responsabilité des chercheurs et des entraîneurs, c'est de développer dans les moyen et long termes, une recherche d'excellence autour de la performance de haut niveau, de faire en sorte que chercheurs, entraîneurs et athlètes se parlent davantage et se comprennent mieux, que chacun soit conscient des contraintes et objectifs respectifs, que de nouveaux financements soient obtenus, pour faire avancer à la fois nos connaissances fondamentales, leurs applications et les résultats de nos sportifs. Il faut aussi dépasser les barrières habituelles entre disciplines, les faire dialoguer, imaginer les transferts qui peuvent se faire du haut niveau vers la pratique sportive et d'activités physiques de tout un chacun, mais aussi être capables de faire le chemin inverse. De nombreux travaux, hors du champ des «sciences du sport», pourraient déjà trouver leur application dans le sport de très haut niveau.

Le temps, souvent long, de la recherche est difficilement compatible avec les exigences de résultats immédiats ou immédiatement applicables pour l'athlète et son entraîneur. Seul un travail de fond, s'inscrivant dans la durée, peut permettre de résoudre cette difficile équation, par-delà les hommes, les compétitions, ou les événements.

Le modèle anglais est souvent pris en exemple. Mais ce modèle, outre ses spécificités structurelles, se caractérise par trois éléments essentiels. Les sommes investies pour la recherche ont été et continuent à être nettement plus significatives que l'effort engagé par la France. Par ailleurs, ces investissements ont débuté bien en amont de la désignation de Londres comme ville hôte, suite aux résultats désastreux des JO d'Atlanta (1996). Enfin, suite à cet électrochoc salutaire ( $36^{\mathrm{e}}$ rang mondial), les résultats sportifs ont été en constante progression près de 10 ans après le lancement des premiers programmes de recherche : 2000 et $2004\left(10^{\mathrm{e}}\right), 2008\left(4^{\mathrm{e}}\right), 2012\left(3^{\mathrm{e}}\right), 2016\left(2^{\mathrm{e}}\right)$ et $2020\left(4^{\mathrm{e}}\right)$.

Les travaux rassemblés dans ce numéro spécial montrent une partie de la diversité et de la richesse des recherches engagées au sein de nos laboratoires. Ils mettent en évidence des forces et des compétences indéniables, et un intérêt croissant pour un domaine sportif souvent laissé pour compte au profit de l'image et du marketing. Mais, faute de quoi les investissements réalisés seront vite obsolètes, il faut d'ores et déjà se projeter dans l'après JO, construire des partenariats durables et efficaces basés sur la confiance et l'intérêt réciproque, poser des questions scientifiques pertinentes où le sport de haut niveau et ses applications trouvent toute leur place sans être nécessairement au cœur du projet scientifique. À ces conditions-là 
seulement, de tels projets pourront être financés par les différents instruments de financement récurrents - et ils sont nombreux - dont on dispose déjà: financements Région, ANR, ANRT, programmes européens, etc. Et à ces conditions-là seulement, la France pourra tenir de manière durable le rang qu'elle ambitionne dans la hiérarchie sportive mondiale.

Vincent NOUGIER

Chargé de Mission Sport au CNRS

Citation de l'article : Nougier V (2021) Numéro spécial: Sciences et technologies innovantes pour l'amélioration des performances sportives de haut niveau. Mov Sport Sci/Sci Mot, 114, 1-2 